S. ISHIHARA AND M. KONISHI

KODAI MATH. J.

3 (1980), 385-396

\title{
COMPLEX ALMOST CONTACT MANIFOLDS
}

\author{
By SHIGERU ISHIHARA AND MARIKO KONISHI
}

\section{$\S 1$. Introduction.}

A complex contact manifold is a complex manifold of odd dimensions $2 m+1$ $(\geqq 3)$ covered by an open covering $\mathcal{A}=\left\{O, O^{\prime}, \cdots\right\}$ consisting of coordinate neighborhoods in such a way that

1) In each $O \in \mathcal{A}$ there is a holomorphic 1-form $w$ satisfying $w \wedge(d w)^{m} \neq 0$ at every point of $O$;

2) If $O \cap O^{\prime} \neq \phi\left(O, O^{\prime} \in \mathcal{A}\right)$, there is a non-vanshing holomorphic function $\lambda$ in $O \cap O^{\prime}$ such that $w^{\prime}=\lambda w$ in $O \cap O^{\prime}$, where $w^{\prime}$ is the holomorphic 1-form given in $O^{\prime}$ (See Kobayashi [3]).

In a previous paper [2] we have studied complex contact structure $\{(O, w) \mid O$ $\in \mathcal{A}\}$ which are induced by fiberings of manifolds with (real) normal contact 3structure and obtained the induced (local) tensor field $G$ of type $(1,1)$ in each $O \in \mathcal{A}$ such that $G^{2}=-I+w \otimes W, w \circ G=0$, where $W$ is the associated vector field of $w$. The local structures $\{(O, G, w, W) \mid O \in \mathcal{A}\}$ are very useful to study curvature properties in the same way as in the real case (See Gray [1]. and Sasaki [4]). In the present paper we first define a system of local structures $\{(O, u, G) \mid O \in \mathcal{A}\}$ which will be called a complex almost contact structure and next show that such a structure induces a complex contact structure defined by Kobayashi [3], when it satisfies a suitable condition, i.e., to be normal.

The authors wish to express their thanks to Professor J.S. Park for his valuable suggestions and remarks.

\section{$\S 2$. Complex almost contact structure.}

Let $M$ be a complex manifold with complex structure $F$ and Hermitian metric $g$ and be covered by an open covering $\mathcal{A}=\left\{O, O^{\prime}, \cdots\right\}$ consisting of coordinate neighborhoods. Then $M$ is called a Complex almost contact manifold if the following conditions $\mathbf{1}$ ) and 2) are satisfied:

1) In each $O \in \mathcal{A}$ there are given a 1 -form $u$ and a tensor field $G$ of type $(1,1)$ such that*)

Received July 23, 1979

*) Functions vector fields, tensor fields and geometric objects we consider are assumed to be differentiable and of class $C^{\infty}$, otherwise stated. Throughout this paper, $X, Y$ and $Z$ denote arbitrary vector fields in $M$. 


$$
\begin{aligned}
& G^{2}=-I+u \otimes U+v \otimes V, \\
& G F=-F G, \quad g(G X, Y)=-g(G Y, X), \\
& G U=0, \quad g(U, U)=1,
\end{aligned}
$$

I being the identity tensor of type $(1,1)$ in $M$, where $U$ and $V$ are respectively the associated vector fields of $u$ and a 1 -form $v$ defined in $O$ by

$$
v=u \circ F \text {, }
$$

i.e., $g(U, X)=u(X)$ and $g(V, X)=v(X)$;

2) If $O \cap O^{\prime} \neq \phi\left(O, O^{\prime} \in \mathcal{A}\right)$, there are functions $a$ and $b$ in $O \cap O^{\prime}$ such that

$$
\begin{aligned}
& u^{\prime}=a u-b v, \quad G^{\prime}=a G-b H, \\
& a^{2}+b^{2}=1 \\
& v^{\prime}=b u+a v, \quad H^{\prime}=b G+a H,
\end{aligned}
$$

in $0 \cap 0^{\prime}$, where $H$ is defined in $O$ by

$$
H=G F
$$

and $\left(u^{\prime}, G^{\prime}\right)$ are the local structure given in $O^{\prime}, v^{\prime}$ and $H^{\prime}$ being defined in $O^{\prime}$ by (2.2) and (2.4) respectively.

The set $\{(O, u, G) \mid O \in \mathcal{A}\}$ is called a complex almost contact structure. In such a case, $M$ is necesarily of odd complex dimensions $2 m+1(\geqq 3)$. For a complex almost structure, we have

$$
\begin{aligned}
& H^{2}=-I+u \otimes U+v \otimes V . \\
& H G=-G H=F+u \otimes V-v \otimes U, \\
& F H=-H F=G, \quad g(H X, Y)=-g(H Y, X), \\
& G V=H U=H V=0, \quad u \circ G=v \circ G=u \circ H=v \circ H=0, \\
& F U=-V, \quad F V=U, \quad u=-v \circ F, \\
& g(V, V)=1, \quad g(U, V)=0
\end{aligned}
$$

as consequences of (2.1), (2.2), (2.3), (2.4) and

We now have

$$
F^{2}=-I, \quad g(F X, Y)=-g(F Y, X) .
$$

THEOREM 1. For a complex almost contact manifold of complex dimensions $2 m+1(\geqq 3)$, the structure group of the tangent bundle of $M$ is reducible to $\left(S_{p}(m) \cdot S_{p}(1)\right) \times U(1)$, where $S_{p}(m) \cdot S_{p}(1)=S_{p}(m) \times S_{p}(1) /\{ \pm 1\}$.

We now put

$$
P=u \otimes U+v \otimes V
$$


locally in each $O \in \mathcal{A}$. Then, as a consequence of (2.3), $P$ determines a global tensor field, which is also denoted by $P$, in $M$ such that $P^{2}=P$. Thus $P$ is a projection tensor of rank 2. The distribution $D$ determined by $P$ is invariant under the action of the complex structure $F$ and called the vertical distribution for brevity. We denote by $B$ the vector bundle over $M$ consisting of all vectors belonging to the vertical distribution $D$.

Let $\nabla$ be the Riemannian connection of $(M, g)$. If we put

$$
P \nabla_{X} U=2 \sigma(X) V, \quad P \nabla_{X} V=-2 \sigma(X) U
$$

in each $O \in \mathcal{A}$, then we get a local 1 -form $\sigma$ in $O$. If $O \cap O^{\prime} \neq \phi\left(O, O^{\prime} \in \mathcal{A}\right)$, we have

$$
2 \sigma^{\prime}=2 \sigma+b^{-1} d a=2 \sigma-a^{-1} d b
$$

in $O \cap O^{\prime}, a$ and $b$ being the functions appearing in (2.3), where $\sigma^{\prime}$ is the local 1 form defined by $(2.4)$ in $O^{\prime}$. Then $\{(2 \sigma, O) \mid O \in \mathcal{A}\}$ defines a linear connection $\theta$ in the vertical vector bundle $B$. The local vector fields defined respectively by

$$
D_{X} U=\nabla_{X} U-2 \sigma(X) V, \quad D_{X} V=\nabla_{X} V+2 \sigma(X) U
$$

are orthogonal to the vertical distribution $D$. If $O \cap O^{\prime} \neq \phi\left(O, O^{\prime} \in \mathcal{A}\right)$, we get by using (2.3) and (2.6)

$$
\left\{\begin{array}{l}
D_{X} U^{\prime}=a D_{X} U-b D_{X} V, \\
D_{X} V^{\prime}=b D_{X} U+a D_{X} V,
\end{array}\right.
$$

where $D_{X} L^{\prime}$ and $D_{X} V^{\prime}$ are defined by (2.8) in $O^{\prime}$.

\section{$\S 3$. Normal complex almost contact structure.}

Let $M$ be a complex almost contact manifold with structure $\{(O, u, G) \mid O \in \mathcal{A}\}$. Denote by $u_{\imath}, v_{\imath}, u^{h}, v^{h}, G_{\imath}{ }^{h}, H_{\imath}{ }^{h}$ and $g_{j i}$ components of $u, v, U, V, G, H, g$ in $O$, respectively. Denoting by $\nabla_{\imath}$ the operator of covariant differentiation in $O$ with respect to the Riemannian connection $\nabla$ of $(M, g)$, we put

$$
D_{j} u_{\imath}=\nabla_{j} u_{i}-2 \sigma_{j} v_{\imath}, \quad D_{j} v_{\imath}=\nabla_{j} v_{i}+2 \sigma_{\jmath} u_{\imath},
$$

where $\sigma=\sigma_{j} d x^{\jmath}$ in $O$. *) Then we obtain

$$
\begin{array}{ll}
u^{k} D_{j} u_{k}=0, & u^{k} D_{\jmath} v_{k}=0, \\
v^{k} D_{j} u_{k}=0, & v^{k} D_{j} v_{k}=0,
\end{array}
$$

where $u^{h}=u_{t} g^{t h}, v^{h}=v_{t} g^{t h}, g^{i n}$ being defined by $\left(g^{i h}\right)=\left(g_{\imath h}\right)^{-1}$, since $D_{X} U$ and $D_{X} V$ are orthogonal to the vertical distribution $D$.

The complex almost contact structure is said to be contact when

*) The indices $h, i, \jmath, k, \cdots, t, s, \cdots$ run over the range $\{1, \cdots, 4 m+2\}$ and the summation convension is used with respect to this system of indices. 


$$
d u-\sigma \wedge v=\hat{G}, \quad d v+\sigma \wedge u=\hat{H},
$$

where local 2 -forms $\hat{G}$ and $\hat{H}$ are defined in $O$ by

$$
\hat{G}(X, Y)=g(G X, Y), \quad \hat{H}(X, Y)=g(H X, Y),
$$

respectively. When the structure is contact,

$$
D_{j} u_{i}-D_{i} u_{j}=2 G_{j i}, \quad D_{j} v_{i}-D_{\imath} v_{j}=2 H_{j i}
$$

hold, where $G_{\jmath i}=G_{\jmath}{ }^{t} g_{t \imath}$, and $H_{j \imath}=H_{\jmath}{ }^{t} g_{t \imath}$ are components of $\hat{G}$ and $\hat{H}$ in $O$ respectively.

We now put in $O$

$$
D_{j} G_{j}{ }^{h}=\nabla_{j} G_{\imath}{ }^{h}-2 \sigma_{j} H_{j}{ }^{h}, \quad D_{j} H_{\imath}{ }^{h}=\nabla_{j} H_{\imath}{ }^{h}+2 \sigma_{j} G_{\imath}{ }^{h},
$$

which are respectively components of local tensor fields of type $(1,2)$ in $O$. If $O \cap O^{\prime} \neq \phi\left(O, O^{\prime} \in \mathcal{A}\right)$, using (2.3) and (2.7), we have in $O \cap O^{\prime}$

$$
D_{\jmath} G_{\imath}{ }^{h}=a D_{j} G_{\imath}{ }^{h}-b D_{j} H_{\imath}{ }^{h}, \quad D_{j} H_{\imath}^{\prime h}=b D_{\jmath} G_{\imath}{ }^{h}+a D_{j} H_{\imath}{ }^{h},
$$

where $D_{\jmath} G_{\imath}^{\prime h}$ and $D_{j} H_{\imath}^{\prime h}$ are defined by (3.5) in $O^{\prime}$.

Next, we define in $O$ local tensor fields $S, T$ and $W$ of type (1.2) respectively by their components as followings:

$$
\begin{aligned}
& S_{k \jmath}{ }^{h}=G_{k}{ }^{t} D_{t} G_{\jmath}{ }^{h}-G_{\jmath}{ }^{t} D_{t} G_{k}{ }^{h}-G_{t}{ }^{h}\left(D_{k} G_{\jmath}{ }^{t}-D_{\jmath} G_{k}{ }^{t}\right) \\
& +2\left(v_{j} H_{k}{ }^{h}-v_{k} H_{j}{ }^{h}\right)+2\left(G_{k j} u^{h}-H_{k j} u^{h}\right), \\
& T_{k \jmath}{ }^{h}=H_{k}{ }^{t} D_{t} H_{\jmath}{ }^{h}-H_{\jmath}{ }^{t} D_{t} H_{k}{ }^{h}-H_{t}{ }^{h}\left(D_{k} H_{\jmath}{ }^{t}-D_{j} H_{k}{ }^{t}\right) \\
& +2\left(u_{j} G_{k}{ }^{h}-u_{k} G_{j}{ }^{h}\right)+2\left(H_{k j} v^{h}-G_{k j} u^{h}\right) . \\
& W_{k \jmath}{ }^{h}=\frac{1}{2}\left[G_{k}{ }^{t} D_{t} H_{\jmath}{ }^{h}+H_{k}{ }^{t} D_{t} G_{\jmath}{ }^{h}-G_{\jmath}{ }^{t} D_{t} H_{k}{ }^{h}-H_{\jmath}{ }^{t} D_{t} G_{k}{ }^{h}\right. \\
& \left.-G_{t}{ }^{h}\left(D_{k} H_{j}{ }^{t}-D_{j} H_{k}{ }^{t}\right)-H_{t}{ }^{h}\left(D_{k} G_{j}{ }^{t}-D_{j} G_{k}{ }^{t}\right)\right] \\
& -\left(u_{j} H_{k}{ }^{h}+v_{j} G_{k}{ }^{h}-u_{k} H_{j}{ }^{h}-v_{k} G_{j}{ }^{h}\right)+2\left(G_{k j} v^{h}+H_{k j} u^{h}\right) .
\end{aligned}
$$

Then we have in $O \cap O^{\prime}$

$$
S^{\prime}=a^{2} S+2 a b W+b^{2} T, \quad T^{\prime}=b^{2} S-2 a b W+a^{2} T,
$$

$$
W^{\prime}=a b(S-T)+\left(a^{2}-b^{2}\right) W,
$$

where $S^{\prime}, T^{\prime}$ and $W^{\prime}$ are defined by (3.7) in $O^{\prime}$. The set $\{S, T, W\}$ of local tensor fields will be called the torsion tensor of the given complex almost contact structure. The equations (3.8) show that if $S=T=W=0$ in $O$, then $S^{\prime}=T^{\prime}=W^{\prime}=0$ in $O \cap O^{\prime}$. When a complex almost contact structure is contact and its torsion 
tensors $S, T$ and $W$ vanish, it is said to be normal.

Proposition 1. If a complex almost contact structure $\{(O, u, v, G, H) \mid O \in \mathcal{A}\}$ is normal, then

$$
G_{j i}=D_{j} u_{\imath}, \quad H_{j i}=D_{j} v_{\imath}
$$

Proof. Since the structure is contact, differentiating exteriorly (3.3), we have

$$
d \hat{G}-\sigma \wedge \hat{H}=-\Omega \wedge v, \quad d \hat{H}+\sigma \wedge \hat{G}=\Omega \wedge u,
$$

where $\Omega=d \sigma$ and $2 \Omega$ is the curvature tensor $\Theta$ of the linear connection $\theta$ induced in the vertical vector bundle $B$. The equations (3.10) are equivalent to

$$
\begin{aligned}
& D_{k} G_{j i}+D_{\jmath} G_{i k}+D_{i} G_{k j}=-\left(\Omega_{k j} v_{i}+\Omega_{j i} v_{k}+\Omega_{i k} v_{j}\right), \\
& D_{k} H_{j i}+D_{j} H_{i k}+D_{i} H_{k j}=\Omega_{k j} u_{i}+\Omega_{j i} u_{k}+\Omega_{i k} u_{\jmath} .
\end{aligned}
$$

where

$$
\begin{array}{ll}
D_{k} G_{j i}=\nabla_{k} G_{j i}-2 \sigma_{k} H_{j i}, & D_{k} H_{j i}=\nabla_{k} H_{j i}+2 \sigma_{k} G_{j i}, \\
\Omega_{j i}=\frac{1}{2}\left(\partial_{j} \sigma_{i}-\partial_{i} \sigma_{j}\right), & \partial_{j}=\frac{\partial}{\partial x \jmath},
\end{array}
$$

$\left(x^{1}, \cdots, x^{4 m+2}\right)$ being local coordinates in $O$.

Putting

$$
\mathfrak{L}_{U} G_{j i}=u^{k} D_{k} G_{j i}+\left(D_{j} u^{k}\right) G_{k i}+\left(D_{\imath} u^{k}\right) G_{j k},
$$

we have from (2.1), (2.2), (2.4), (2.5) and (3.11)

$$
\mathfrak{S}_{U} G_{j i}=u^{k}\left(D_{k} G_{j i}+D_{j} G_{i k}+D_{i} G_{k \jmath}\right)=-u^{k}\left(\Omega_{k j} v_{i}+\Omega_{i k} v_{\jmath}\right) .
$$

On the other hand, since $S_{k_{3}}{ }^{h}=0$, transvecting the first equation of (3.7) with $G_{h}{ }^{t} u^{j}$, we get

$$
\begin{aligned}
& 0=S_{k j}{ }^{h} G_{h}{ }^{t} u^{j} \\
& =-G_{k}{ }^{r}\left(D_{r} u^{j}\right) G_{j}{ }^{h} G_{h}{ }^{t}+\left(G_{r}{ }^{h} G_{h}{ }^{t}\right)\left(D_{k} u^{j}\right) G_{j}{ }^{r}+\left(G_{r}{ }^{h} G_{h}{ }^{t}\right) u^{\jmath} D_{j} G_{k}{ }^{r} \\
& =-\left(u^{j} D_{j} G_{k}{ }^{t}-G_{k}{ }^{2} D_{\imath} u^{t}+G_{j}{ }^{t} D_{k} u^{j}\right) \\
& +G_{k}^{r} u^{t} u^{j}\left(D_{j} u_{r}-D_{r} u_{j}\right)+G_{k}^{r} v^{t} u^{j}\left(D_{j} u_{r}-D_{r} u_{j}\right) .
\end{aligned}
$$

Next, we put

$$
\mathfrak{乏}_{U} G_{\jmath}{ }^{t}=u^{k} D_{k} G_{\jmath}{ }^{t}-G_{\jmath}{ }^{k} D_{k} u^{t}+G_{k}{ }^{t} D_{j} u^{k} .
$$

Then, using (2.1), (2.2), (2.4), (2.5), (3.4) and (3.13), we have

from which

$$
\mathfrak{L}_{U} G_{j}{ }^{t}=0,
$$




$$
\begin{aligned}
0 & =\left(\mathfrak{Q}_{U} G_{\jmath}{ }^{t}\right) g_{t \imath} \\
& =\left(u^{k} D_{k} G_{j}{ }^{t}-G_{\jmath}{ }^{k} D_{k} u^{t}+G_{k}{ }^{t} D_{j} u^{k}\right) g_{t \imath} \\
& =u^{k} D_{k} G_{j i}-G_{\jmath}{ }^{k}\left(2 G_{k i}+D_{i} u_{k}\right)+G_{k i} D_{j} u^{k} \\
& =\left(u^{k} D_{k} G_{j i}+G_{k i} D_{j} u^{k}+G_{j k} D_{i} u^{k}\right)-2 G_{\jmath}{ }^{k}\left(G_{k i}+D_{\imath} u_{k}\right) \\
& =\mathfrak{Q}_{U} G_{j i}-2 G_{\jmath}{ }^{k}\left(G_{k i}+D_{i} u_{k}\right) .
\end{aligned}
$$

Substituting (3.12) into this, we obtain

$$
2 G_{j}{ }^{k}\left(G_{k i}+D_{i} u_{k}\right)=-u^{k}\left(\Omega_{k j} v_{\imath}+\Omega_{i k} v_{j}\right),
$$

from which, transvecting $v^{\nu}$,

$$
u^{k} \Omega_{k \imath}=\left(u^{k} \Omega_{k j} v^{j}\right) v_{\imath} .
$$

Thus, we have from (3.14) and (3.15)

and hence, using (3.2),

$$
G_{j}{ }^{k}\left(G_{k i}+D_{i} u_{k}\right)=0
$$

$$
G_{k i}+D_{i} u_{k}=0 \text {. }
$$

Consequently, we get

$$
G_{j i}=D_{j} u_{\imath}=-D_{i} u_{\jmath} .
$$

Similarly, we obtain

$$
v^{k} \Omega_{k \imath}=\left(v^{k} \Omega_{k j} u^{j}\right) u_{\imath}
$$

and

$$
H_{j \imath}=D_{j} v_{i}=-D_{\imath} v_{\jmath} .
$$

Proposition 2. A complex almost contact structure is normal if and only if it is contact and

$$
\begin{aligned}
& D_{j} G_{\imath}{ }^{h}=\delta_{j}^{h} u_{\imath}-g_{j i} u^{h}+F_{j}{ }^{h} v_{\imath}-F_{j i} v^{h}, \\
& D_{j} H_{\imath}{ }^{h}=\delta_{j}^{h} v_{i}-g_{j i} v^{h}-F_{j}{ }^{h} u_{i}+F_{j i} u^{h},
\end{aligned}
$$

where $F_{j}{ }^{h}$ are components of the complex structure $F$ and $F_{j i}=F_{\jmath}{ }^{t} g_{t \imath}$.

Proof. First, the given structure is assumed to be normal. Since it is contact, we have from (3.11)

$$
D_{k} G_{j t}+D_{j} G_{t k}+D_{t} G_{k j}=-\left(\Omega_{k j} v_{t}+\Omega_{j t} v_{k}+\Omega_{t k} v_{j}\right)
$$

from which, transvecting $v^{t}$,

$$
\begin{aligned}
v^{t} D_{t} G_{k \jmath}-G_{\jmath}{ }^{t} D_{k} v_{t}+G_{k}{ }^{t} D_{\jmath} v_{t} \\
=-\left(\Omega_{k \jmath}+\left(v^{t} \Omega_{\jmath t}\right) v_{k}+\left(v^{t} \Omega_{t k}\right) v_{\jmath}\right) .
\end{aligned}
$$


On the other hand, since $S_{k j}{ }^{h}=0$, transvecting the first equation of (3.7) with $G_{h}{ }^{t} v^{\jmath}$ and using (2.1), (2.2), (2.4), (2.5) and (3.2), we have

$$
\begin{aligned}
& 0=S_{k j}{ }^{h} G_{h}{ }^{t} v^{\jmath} \\
& =\left\{G_{k}{ }^{r} G_{h}{ }^{t} D_{r} G_{j}{ }^{h}-\left(G_{r}{ }^{h} G_{h}{ }^{t}\right)\left(D_{k} G_{j}{ }^{r}-D_{j} G_{k}{ }^{r}\right)\right\} v^{\jmath}-2\left(F_{k}{ }^{t}+u_{k} v^{t}-v_{k} u^{t}\right) \\
& =-G_{k}{ }^{r}\left(G_{j}{ }^{h} G_{h}{ }^{t}\right) D_{r} v^{\jmath}-\left(\delta_{r}^{t}-u_{r} u^{t}-v_{r} v^{t}\right)\left(G_{j}{ }^{r} D_{k} v^{\jmath}+v^{\jmath} D_{j} G_{k}{ }^{r}\right) \\
& -2\left(F_{k}{ }^{t}+u_{k} v^{t}-v_{k} u^{t}\right) \\
& =-\left(v^{j} D_{j} G_{k}{ }^{t}-G_{k}{ }^{r} D_{r} v^{t}+G_{j}{ }^{t} D_{k} v^{j}\right)-2\left(F_{k}{ }^{t}+u_{k} v^{t}-v_{k} u^{t}\right)
\end{aligned}
$$

and hence

$$
v^{j} D_{j} G_{k}{ }^{t}-G_{k}{ }^{r} D_{r} v^{t}+G_{j}{ }^{t} D_{k} v^{j}=-2\left(F_{k}{ }^{t}+u_{k} v^{t}-v_{k} u^{t}\right) .
$$

This implies together with (3.16), (3.17) and (3.20)

$$
\Omega_{k j}=2 F_{k j}+(2-\alpha)\left(u_{k} v_{j}-v_{k} u_{j}\right),
$$

where $\alpha=\Omega_{k j} v^{k} u^{j}$.

We now put $S_{k j i}=S_{k \jmath}{ }^{t} g_{t \imath}$. Then, using (3.9), (3.11) and (3.21), we have

$$
\begin{aligned}
& 0=S_{k j i} \\
& =G_{k}{ }^{r} D_{r} G_{j i}-G_{j}{ }^{r} D_{r} G_{k i}-G_{r i}\left(D_{k} G_{j}{ }^{r}-D_{j} G_{k}{ }^{r}\right) \\
& +2\left(v_{j} H_{k i}-v_{k} H_{j i}+G_{k j} u_{i}-H_{k \jmath} v_{\imath}\right) \\
& =G_{k}{ }^{r}\left(D_{r} G_{j i}-D_{j} G_{r i}\right)-G_{j}{ }^{r}\left(D_{r} G_{k i}-D_{k} G_{r i}\right) \\
& +v_{j} H_{k i}-v_{k} H_{j i}+u_{k} G_{j i}-u_{j} G_{k i}-4 H_{k j} v_{\imath} \\
& =-G_{k}{ }^{r}\left(D_{i} G_{r j}+2 F_{r j} v_{i}+2 F_{\imath r} v_{j}\right)+G_{j}^{r}\left(D_{i} G_{r k}+2 F_{r k} v_{i}+2 F_{\imath r} v_{k}\right) \\
& +v_{j} H_{k i}-v_{k} H_{j i}+u_{k} G_{j i}-u_{j} G_{k i}-4 H_{k j} v_{\imath} \\
& =-D_{i}\left(G_{k}^{r} G_{r j}\right)+2 G_{j}{ }^{r} D_{i} G_{r k}-v_{\jmath} H_{k i}+v_{k} H_{j i}-u_{k} G_{\imath j}-u_{j} G_{k \imath} \\
& =2\left(G_{j}^{r} D_{i} G_{r k}-H_{\imath j} v_{k}-G_{\imath j} u_{k}\right) \text {, }
\end{aligned}
$$

from which

$$
G_{\jmath}^{r} D_{i} G_{r k}=u_{k} G_{\imath \jmath}+v_{k} H_{\imath \jmath}
$$

Thus, transvecting this with $G_{t}{ }^{3}$, we get

$$
D_{j} G_{t k}=u_{t} g_{j k}-u_{k} g_{i t}+v_{t} F_{i k}-v_{k} F_{i t}
$$

and hence the first equation of (3.19). Similarly, we obtain the second equation of (3.19).

Conversely, the given complex almost contact structure is assumed to satisfy (3.19). Transvecting the first equation of (3.19) with $u^{2}$, we get 


$$
\left(D_{j} G_{\imath}{ }^{h}\right) u^{\imath}=\delta_{j}^{h}-u_{j} u^{h}-v_{j} v^{h} .
$$

Since $G_{\imath}{ }^{h} u^{\imath}=0$, we have

from which

$$
G_{i}{ }^{h}\left(D_{j} u^{i}\right)=-\delta_{j}^{h}+u_{j} u^{h}+v_{j} v^{h},
$$

$$
D_{j} u^{h}=G_{j}{ }^{h} \text {, i. e., } \quad D_{j} u_{i}=G_{j i} .
$$

This means

$$
D_{j} u_{i}-D_{i} u_{j}=2 G_{j i}
$$

Similarly, we obtain

$$
D_{j} v_{i}-D_{\imath} v_{j}=2 H_{j i} .
$$

From (3.22) and (3.23) we see that the given structure is contact. Next, using (3.19), we can easily verify $S=T=W=0$. Consequently, in this case the given structure is normal.

Q. E. D.

PROPOSITION 3. If a complex almost contact structure is normal, the pair $(F, g)$ is a Kahlerian structure, i.e., $\nabla F=0$.

Proof. Using $H_{\imath}{ }^{h}=G_{t}{ }^{h} F_{\imath}{ }^{t}$, we have

$$
D_{j} H_{\imath}{ }^{h}=\left(D_{j} G_{t}{ }^{h}\right) F_{\imath}{ }^{t}+G_{t}{ }^{h}\left(\nabla_{j} F_{\imath}{ }^{t}\right),
$$

from which, substituting (3.19),

$$
G_{t}{ }^{h}\left(\nabla_{j} F_{\imath}{ }^{t}\right)=0 \text {. }
$$

Thus, we get

$$
-\nabla_{j} F_{\imath}{ }^{h}+\left(u_{t} \nabla_{j} F_{\imath}{ }^{t}\right) u^{h}+\left(v_{t} \nabla_{j} F_{\imath}{ }^{t}\right) v^{h}=0,
$$

from which, using (2.1) and Proposition 1,

$$
\nabla_{j} F_{\imath}{ }^{h}=0 . \quad \text { Q.E. D. }
$$

Proposition 4. For a complex almost contact structure, which is normal,

$$
\Omega_{\jmath k}=2 F_{j k},
$$

i.e., the curvature form $\Theta$ of the linear connection $\theta$ induced $2 n$ the vertical vector bundle $B$ is given by

$$
\Theta(X, Y)=2 g(F X, Y) .
$$

Proof. Since $\Omega=d \sigma$, we have $d \Omega=0$. Thus, using (3.21), we have

$$
(2-\alpha)(d u \wedge v-u \wedge d v)+d \alpha \wedge u \wedge v=0
$$

because $\nabla F=0$. Substituting into this 


$$
d u \wedge v=\hat{H} \wedge v, \quad u \wedge d v=u \wedge \hat{G}
$$

which are direct consequences of (3.9), we obtain

$$
(2-\alpha)(\hat{H} \wedge v-u \wedge \hat{G})+d \alpha \wedge u \wedge v=0 .
$$

Taking account of $(\hat{H} \wedge v)(X, U, V)=(u \wedge \hat{G})(X, U, V)=0$, we have from (3.22)

$$
d \alpha=(U \alpha) u+(V \alpha) v
$$

from which and (3.22)

$$
(2-\alpha)(\hat{H} \wedge u-u \wedge \hat{G})=0
$$

and hence $\alpha=2$. Thus, substituting $\alpha=2$ into (3.21), we get

$$
\Omega_{j k}=2 F_{j k} \text {. }
$$

Q.E.D.

$\S 4$. Curvature properties. In this section, let $(M, G, F)$ be a complex manifold of (real) dimension $n$ with complex almost contact structure $\{(O, u, v, G, H)\}$ which is normal. Using (3.19) and Ricci formulas gives

$$
\begin{aligned}
& -K_{k j i}{ }^{s} u_{s}=u_{j} g_{k i}-u_{k} g_{j i}+v_{j} F_{k i}-v_{k} F_{j i}-2 v_{i} F_{k j}+2 \Omega_{k j} v_{\imath}, \\
& -K_{k j i}{ }^{s} v_{s}=v_{j} g_{k i}-v_{k} g_{j i}-u_{j} F_{k i}+u_{k} F_{j i}+2 u_{i} F_{k j}-2 \Omega_{k j} u_{\imath},
\end{aligned}
$$

$K_{k j i}{ }^{h}$ being components of the curvature tensor $K$ of $(M, g, F)$, where $F_{j i}=F_{j}{ }^{h} g_{j i}$ and

$$
\Omega_{j i}=\frac{1}{2}\left(\partial_{\jmath} \sigma_{\imath}-\partial_{i} \sigma_{j}\right) .
$$

Next, (3.19) and Ricci formulas imply

$$
K_{k j t}{ }^{h} G_{\imath}{ }^{t}-K_{k j i}{ }^{t} G_{t}{ }^{h}=G_{k i} \delta_{j}^{h}-G_{j i} \delta_{k}^{h}+G_{k}{ }^{h} g_{j i}+G_{j}{ }^{h} g_{k \imath}
$$

$$
+H_{k i} F_{j}{ }^{h}-H_{j i} F_{k}{ }^{h}-H_{k}{ }^{h} F_{j i}+H_{j}{ }^{h} F_{k i}+2 \Omega_{k j} H_{i}{ }^{h} .
$$

Changing in (4.3) the index $h$ to $s$ and then transvecting $G_{s h}\left(=G_{s}{ }^{t} g_{t h}\right)$, we have by means of $(4.1)$

$$
K_{k j i h}-K_{k j t s} G_{\imath}{ }^{t} G_{h}{ }^{s}=\left(G_{k i} G_{j h}-G_{j i} G_{k h}\right)+\left(H_{k i} H_{j h}-H_{j i} H_{k h}\right)
$$

$$
-\left(F_{k i} F_{j h}-F_{j i} F_{k h}\right)-\left(g_{k i} g_{j h}-g_{j i} g_{k h}\right)-2 \Omega_{k j} F_{i h},
$$

where $K_{k j i h}=K_{k j i}{ }^{s} g_{s h}$ and $H_{j i}=H_{j}^{s} g_{s i}$.

Since $(M, g, F)$ is Kahlerian, we obtain

$$
K_{k j i h}=K_{k j t s} F_{\imath}{ }^{t} F_{h}{ }^{s} \text {. }
$$

Then, transvecting $G^{i n}\left(=g^{\imath s} G_{s}{ }^{n}\right)$ with (4.5) gives 


$$
K_{k j i h} G^{\imath h}=0 \text {. }
$$

Similarly, we get

$$
K_{k j i h} H^{2 h}=0 .
$$

On the other hand, we have

$$
K_{k t s h} G^{t s}=\frac{1}{2}\left(K_{k t s h}-K_{k s t h}\right) G^{t s}=-\frac{1}{2} K_{k h t s} G^{t s},
$$

where we used the identity $K_{k j i h}+K_{j i k h}+K_{i k j h}=0$. Thus (4.6) and (4.8) give

$$
K_{k t s h} G^{t s}=0 .
$$

If we transvect $g^{j i}$ with (4.4), then we have by using (4.9)

$$
K_{k h}=(n-2) g_{k h}+2 \Omega_{k s} F_{h}{ }^{s} .
$$

Next, transvecting $F^{j i}\left(=g^{\jmath s} F_{s}{ }^{i}\right)$ with (4.4), then we have by using (4.7)

$$
K_{k j i h} F^{j i}=(n-2) F_{k h}+2 \Omega_{k h} .
$$

Transvecting $F^{i h}$ with (4.4) gives

$$
K_{k j i h} F^{i h}=-(n+2) \Omega_{k \jmath} .
$$

On the other hand, (4.5) implies

$$
K_{k j}=K_{k t s h} F^{t s} F_{\jmath}{ }^{h}=-\frac{1}{2} K_{k h t s} F^{t s} F_{\jmath}{ }^{h},
$$

which is obtained in the same way as (4.8) done. This equation and (4.12) imply

$$
K_{k j}=\frac{n+2}{2} \Omega_{k s} F_{j}^{s} \text {. }
$$

If we substitute (4.13) in (4.10), we have

$$
\Omega_{k j}=2 F_{k \jmath},
$$

which gives Proposition 4. Substituting (4.14) into (4.13), we have

$$
K_{k J}=(n+2) g_{k J}
$$

and hence

$$
K=K_{k j} g^{k \jmath}=n(n+2),
$$

where $K$ denotes the scalar curvature of $(M, g, F)$. Thus we have form (4.15) and (4.16) 
THEOREM 2. If a complex manifold $(M, F, g)$ with Hermitian metric $g$ admits a complex almost contact structure, which is normal, then $(M, F, g)$ is an Einstern Kahlerian space with scalar curvature $n(n+2)$, where $\operatorname{dim} M=n(\geqq 3)$. If moreover $M$ is complete, then $M$ is compact.

We now take complex coordinates $\left(Z^{1}, \cdots, Z^{2 m+1}\right)$ in $O$, such that $F$ has components of the form

$$
F_{\imath}^{h}=\left(\begin{array}{cc}
\sqrt{-1} \delta_{\lambda}^{\kappa} & 0 \\
0 & -\sqrt{-1} \delta_{\frac{\kappa}{\lambda}}^{\bar{\kappa}}
\end{array}\right) .
$$

Putting

$$
\pi=u+\sqrt{-1} v,
$$

we see that $\pi$ is a complex 1 -form of type $(1,0)$, i. e. $\pi=\pi_{\kappa} d Z^{\kappa}$. Since (4.14) holds, we can find a holomorphic 1 -form $w=f \pi$ in $O$, where $f$ is a function defined in $O$, such that $w \wedge(d w)^{m} \neq 0$ (for proof see [2]). Thus we have

THEOREM 3. If a complex manifold $M$ with Hermitian metric $g$ admits a complex almost contact structure, which is normal, there is in $M$ a complex contact structure.

Under the same assumption as in Theorem 3, using Proposition 1, we see that $[U, V]$ belongs to $D$, i. e. that the vertical distribution $D$ is integrable. A maximal integral submanifold of $D$ will be called a fibre.

Proposition 5. Under the same assumption as in Theorem 3, the vertical distribution $D$ is integrable and every flbre is a totally geodesic submanifold with complex dimension 1 and with constant curvature 4 . If moreover $M$ is complete, every fibre is a 2-dimensional sphere with curvature 4.

Proof. Proposition 1 implies

$$
\nabla_{U} U=2 \sigma(U) V, \quad \nabla_{V} U=2 \sigma(V) V, \quad \nabla_{U} V=-2 \sigma(U) U, \quad \nabla_{V} V=-2 \sigma(V) U,
$$

which show that every fiber is totally geodesic. Since $F U=-V, F V=U$, every fiber is a complex submanifold of complex dimension 1. Next, using (4.1), we have $K_{k, i n} V^{k} U^{j} V^{\imath} U^{h}=4$, which means that every fiber has constant curvature 4 .

Q.E.D.

Remark. For a complex almost contact manifold $(M, F, g)$ with structure $\{(O, u, G) \mid O \in \mathcal{A}\}$, the Hermitian manifold $(M, F, g)$ is assumed to be Kahlerian. Then the local tensor field $G$ has components satisfying*)

*) The indices $\lambda, \mu, \nu, \tau \cdots$ run over the range $\{1, \cdots, 2 m+1\}$ and the summation convension is used with respect to this system of indices. 


$$
G_{\mu}^{\lambda}=0, \quad G_{\bar{\mu}}^{\bar{\lambda}}=0
$$

with respect to complex coordinates $\left(Z^{1}, \cdots, Z^{2 m+1}\right)$ in each $O \in \mathcal{A}$. The local tensor fields $S, T$ and $W$ have respectively components satisfying

$$
\begin{aligned}
& S_{\nu \mu}^{\lambda}=0 \text {, } \\
& S_{\nu \bar{\mu}}{ }^{\lambda}=G_{\nu}{ }^{\bar{\tau}} D_{\bar{\tau}} G_{\bar{\mu}}^{\lambda}+G_{\bar{\tau}}^{\lambda} D_{\bar{\mu}} G_{\nu}^{\bar{\tau}}-2 u_{\nu} G_{\bar{\mu}}^{\lambda}, \\
& S_{\bar{\nu} \bar{\mu}^{\lambda}}=G_{\bar{\nu}}^{-\tau} D_{\bar{\tau}} G_{\bar{\mu}}^{\lambda}-G_{\bar{\mu}}^{-\tau} D_{\tau} G_{\bar{\nu}}^{\lambda}+2\left(u_{\bar{\nu}} G_{\bar{\mu}}^{\lambda}-u_{\bar{\mu}} G_{\bar{\nu}}^{-\tau}\right)+4 G_{\bar{\nu} \mu} u^{\lambda} \text {; } \\
& T_{\nu \mu}^{\lambda}=0, \quad T_{\overline{\nu \mu}}^{\lambda}=S_{\nu \bar{\mu}}^{\lambda}, \quad T_{\overline{\nu \mu}}^{\lambda}=-S_{\overline{\nu \mu}}^{\lambda} ; \\
& W_{\nu \mu}^{\lambda}=0, \quad W_{\nu \bar{\mu}}^{\lambda}=0, \quad W_{\overline{\nu \mu}}^{\lambda}=-\sqrt{-1} S_{\nu \bar{\mu}}^{\lambda} .
\end{aligned}
$$

The equation

$$
G^{2}=-I+u \otimes U+v \otimes V
$$

given in (2.1) is equivalent to

$$
G_{\bar{\tau}}^{\lambda} G_{\mu} \bar{\tau}=-\delta_{\mu}^{\lambda}+2 u_{\mu} u^{\lambda} .
$$

The equations (3.9) are equivalent to

$$
G_{\mu \lambda}=D_{\mu} u_{\lambda}, \quad 0=D_{\mu} u_{\bar{\lambda}} .
$$

The $D_{\jmath} G_{\imath}{ }^{h}$ satisfies the identities

$$
D_{\nu} G_{\mu}{ }^{\lambda}=0, \quad D_{\nu} G_{\mu}{ }^{i}=0
$$

and the equations (3.19) are equivalent to

$$
D_{\nu} G_{\bar{\mu}}^{\lambda}=2\left(\delta_{\mu}^{\lambda} u_{\bar{\mu}}-g_{\nu \bar{\mu}} u^{\lambda}\right), \quad D_{\bar{\nu}} G_{\bar{\mu}}^{\lambda}=0 .
$$

\section{BIBLIOGRAPHY}

[1] Gray, J.W., Some global properties of contact structures. Ann. Math. 69 (1959), 421-450.

[2] IshiHARA, S., AND M. Konishi, Real contact 3-structure and complex contact structure, Southeast Asian Bulletin of Mathematics, 3 (1979), 151-161.

[3] Kobayashi, S. Remarks on complex contact manifold, Proc. Amer. Math. Soc. vol. 10 (1959), 164-167.

[4] SASAKI, S., Almost contact manifolds I, Lecture note, Tôhoku Univ., 1965.

Tokyo Institute of Technology 